\title{
Dengue fever masquerading as hypokalemic paralysis: a case report
}

\author{
Rajesh Rajput, Deepak Jain, Ashima Mittal, Anoop Kumar, Vaibhav Pathak \\ Department of Medicine, Pt. B.D. Sharma University of Health Sciences, Rohtak, India
}

\begin{abstract}
Dengue fever is a mosquito-borne arboviral disease endemic in tropical countries. The spectrum of presentation of dengue fever ranges from classical flu like illness to dengue hemorrhagic fever and dengue shock syndrome. It is usually regarded as a non-neurotropic virus and neurological manifestations of dengue are rare and hypokalemic quadriparesis is even rarer. Here we present a case report of a patient who presented with hypokalemic quadriparesis due to dengue.
\end{abstract}

\section{Introduction}

Dengue fever is a mosquito-borne arboviral disease endemic in tropical countries. It is caused by four serotypes (DEN 1-4). Spectrum of this disease ranges from mild self-limiting fever to potentially fatal severe multiorgan dysfunction. Neurological manifestations of dengue are rare and hypokalemic quadriparesis is even rarer. Here we present a case report of a patient who presented with hypokalemic quadriparesis due to dengue.

\section{Case Report}

A 25-year-old male presented to emergency with fever of 4 days duration and weakness of all limbs for one day. Fever was high grade, continuous, associated with chills and rigors. Weakness of limbs was sudden in onset first involving lower limbs and then ascended to involve the upper limbs over a period of 4 to 5

Correspondence: Deepak Jain, Department of Medicine, Pt. B.D. Sharma University of Health Sciences, Rohtak-124001 (Haryana), India.

Tel.: +91.9416147887. E-mail: jaindeepakdr@gmail.com

Key words: Dengue fever; quadriparesis; hypokalemia; mosquito borne disease.

Conflict of interest: the authors declare no potential conflict of interest.

Received for publication: 15 August 2016.

Revision received: 27 October 2016.

Accepted for publication: 23 May 2017.

This work is licensed under a Creative Commons Attribution NonCommercial 4.0 License (CC BY-NC 4.0).

(C) Copyright R. Rajput et al., 2017

Licensee PAGEPress, Italy

Italian Journal of Medicine 2017; 11:393-395

doi:10.4081/itjm.2017.767 hours. There was no history of any sensory symptoms like paresthesia, numbness or tingling sensation, bowel or bladder problems, band like sensation, any root pains or cranial nerve involvement. There was no history of loose stools, trauma and recent vaccination. No prior history of any similar episode of sudden onset weakness of limbs was present. Family history was not significant. There was no history of any chronic illness like hypertension, diabetes or thyroid dysfunction and indigenous drug intake.

On examination, patient was conscious, oriented and afebrile. His blood pressure was 130/90 mm Hg and pulse rate was $92 / \mathrm{min}$. His face was plethoric and erythematous rashes were present predominantly over the trunk. On neurological examination, power in proximal muscles of upper and lower limbs was grade $3 / 5$ and deep tendon reflexes were absent. The power in distal muscles was $5 / 5$. Plantar reflex was silent in both lower limbs. There were no signs of cranial nerve, sensory, bowel and bladder dysfunction. Abdomen was diffusely tender but there was no organomegaly. Cardiovascular and respiratory system examination were unremarkable.

On laboratory examination, complete hemogram showed hemoconcentration with hemoglobin of 18.2 $\mathrm{gm} \%$ and hematocrit of $54 \%$. Total leukocyte count was low (3000/cu mm) with differential count of 55\% polymorphs, $42 \%$ lymphocytes, $3 \%$ monocytes. Thrombocytopenia was also evident with platelets count of 1.0 lakh. His serum potassium was low (2.2 $\mathrm{mg} / \mathrm{dL}$ ), while serum sodium and chloride levels were normal. Liver function tests were mildly deranged with aspartate and alanine aminotransferase levels of 64/52 U/L. Renal function tests were within normal limit. Arterial blood gas revealed $\mathrm{pH}$ of 7.38 , bicarbonate levels of $22 \mathrm{mEq} / \mathrm{L}$ and anion gap of 8 . Thyroid profile, blood sugar and creatinine kinase levels were also normal. Urinary sodium and potassium were within normal range.

Dengue NS1 antigen was positive by enzymelinked immunosorbent assay method and im- 
munoglobulin (Ig) M antibody for Dengue virus was positive on $4^{\text {th }}$ day. There was no evidence of malarial parasite on peripheral blood film. Rapid test for malarial antigen, IgM antibodies for leptospira and scrub typhus were negative. Urine complete examination was normal with normal $\mathrm{pH}$ and electrolytes. Chest X-ray was normal while abdomen ultrasonography revealed mild gall bladder edema. Cerebrospinal fluid examination was normal. Electrocardiogram showed U waves suggestive of hypokalemia (Figure 1). Nerve conduction velocity studies showed decreased amplitude of compound muscle action potential of bilateral median, ulnar, radial, axillary, tibial and common peroneal nerve with normal conduction velocity.

A diagnosis of dengue fever with acute hypokalemic paralysis was made. Patient was treated with potassium infusion in normal saline with gradual improvement in power of all limbs. On $2^{\text {nd }}$ day patient's potassium level was normal. Patient was monitored for bleeding from any site. Patient was discharged in a stable condition with no residual muscle weakness and with normal electrolytes, renal and liver function.

\section{Discussion}

Dengue fever is acute viral illness caused by arbovirus of flaviviridae family and transmitted by Aedes aegypti mosquito. The spectrum of presentation of dengue fever ranges from classical flu like illness to dengue hemorrhagic fever and dengue shock syndrome. In 2009 the World Health Organization (WHO) classification system, dengue fever was divided into dengue with or without warning signs and severe dengue. ${ }^{1}$ However in 2011 revised WHO guidelines, dengue was divided into dengue fever, dengue hemorrhagic fever without shock or with shock and expanded dengue syndrome. Expanded dengue syndrome is a new entity added to the classification system to incorporate a wide spectrum of unusual manifestations of dengue infection affecting various organ systems including gastrointestinal, hepatic, neurological, pulmonary and renal systems. ${ }^{2}$ It is usually regarded as a non-neurotropic virus. However, there are recent reports on neuroinvasion of Dengue virus infection. The neurological complication in dengue infection has been hypothesized by three possible mechanisms concerned with neurotropism leading

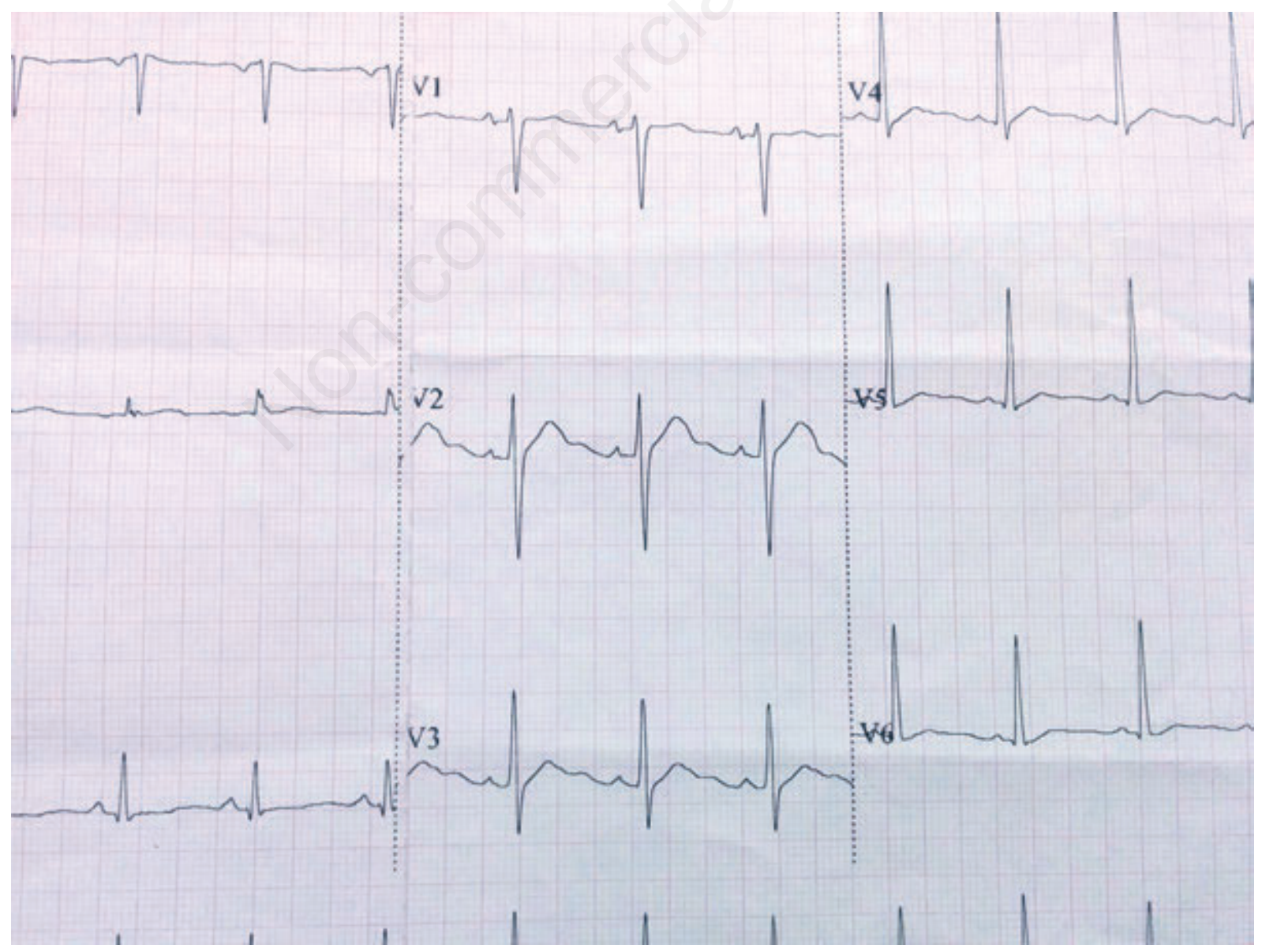

Figure 1. Electrocardiogram showing $U$ waves suggestive of hypokalemia. 
to encephalitis, meningitis and myelitis, systemic complication resulting in encephalopathy, stroke, hypokalemic paralysis and post infectious immune mediated acute disseminated encephalopathy, GuillainBarré syndrome. ${ }^{3}$ Hypokalemia is a known cause of pure motor weakness. Hypokalemia in dengue infection is not very uncommon. Infection-induced hypokalemia is reported in $28 \%$ of serology proven dengue case. ${ }^{4}$ But hypokalemia in tune to presenting as quadriparesis is very rare. There is paucity of literature documenting association of motor weakness in dengue infection. Koshy et al. conducted a study on 799 patients of dengue fever. Out of these, 21 patients $(2.63 \%)$ were having neurological presentation including 7 cases of hypokalemia with quadriparesis. ${ }^{5}$

Our patient had hypokalemic paralysis, which improved after potassium supplementation. Other causes of hypokalemia such as urinary potassium wasting syndrome, renal tubular acidosis, thyrotoxicosis and gastrointestinal loss of potassium were ruled out in our case with relevant investigations and urinary examination. Guillain-Barré syndrome was not considered as a differential because of presentation of fever at the time of presentation and rapid response to potassium supplementation. Familial periodic paralysis was also unlikely because of negative family history, age of presentation and this being the first episode.

Probable mechanism of hypokalemia in dengue fever could be vomiting and loose stools resulting in potassium loss, transcellular shifts causing rapid intracellular shifting (as a result of increase in stress hormones like catecholamines, rapid cell growth, transient renal abnormality including potassium wasting states), and increased renin levels due to decreased intravascular volume because of capillary leakage in critical stage of dengue. ${ }^{6,7}$

Quadriparesis has also been reported in other tropical infections like leptospirosis, chikungunya fever and malaria. ${ }^{8-10}$ In our patient all of these infections were excluded with appropriate investigations.

\section{Conclusions}

Areflexic hypokalemic quadriparesis with sudden onset of fever could be a manifestation of dengue fever. Neurological manifestation in form of expanded dengue syndrome are under recognized and underreported. In tropical countries like India especially in rainy seasons, clinicians should be aware of such a presentation of dengue, which is one of the most common arthropod borne diseases and should exercise high index of suspicion to diagnose this treatable entity.

\section{References}

1. World Health Organization (WHO). Dengue: Guidelines for diagnosis, treatment, prevention and control. New edition. Geneva: World Health Organization; 2009.

2. World Health Organization (WHO). Comprehensive guidelines for prevention and control of dengue and dengue hemorrhagic fever. Revised and expanded edition. Geneva: World Health Organization - Regional Office for South-East Asia; 2011.

3. Murthy J. Neurological complication of dengue infection. Neurol India 2010;58:581-4.

4. Ying RS, Tang XP, Zhang FC, et al. [Clinical characteristics of the patients with dengue fever seen from 2002 to 2006 in Guangzhou.] Zhonghua Shi Yan He Lin Chuang Bing Du Xue Za Zhi 2007;21:123-5. [In Chinese].

5. Koshy JM, Joseph DM, John M, et al. Spectrum of neurological manifestations in Dengue virus infection in Northwest India. Trop Doc 2012;42:191-4.

6. Jha S, Ansari MK. Dengue infection causing acute hypokalemic quadriparesis. Neurol India 2010;58:592-4.

7. Gulati S. Dengue infection causing acute hypokalemic quadriparesis. Neurol India 2011;59:143.

8. Morgan AG, Cawich F. Ascending polyneuropathy in leptospirosis: a case study. Ann Trop Med Parasitol 1980;74:567-8.

9. Rampal, Sharda M, Meena H. Hypokalemic paralysis following Chikungunya fever. J Assoc Physicians India 2007;55:598.

10. Kanjalkar M, Karnad DR, Narayana RV, Shah PU. Guillian Barre syndrome following malaria. J Infect 1999; 38:48-50. 